\title{
Lock-in contact thermography on solar cells - comparison with IR-measurements
}

\author{
by O. Breitenstein ${ }^{1}, \mathrm{~K}$. Iwig ${ }^{1}$, I. Konovalov', and D. Wu ${ }^{3}$ \\ ${ }^{1}$ Max Planck Institute of Microstructure Physics, Weinberg 2, D-06120 Halle, Germany; ${ }^{2}$ Shevchenko \\ University, Radiophysical Faculty, 64 Vladimirskaja, Kiev-33, UA-252033 Ukraine; ${ }^{3}$ Universitat Stuttgart, \\ IKP, Pfaffenwaldring 32, D-70569 Stuttgart, Germany
}

\begin{abstract}
Since all internal forward currents flowing during operation of a photovoltaic solar cell represent losses in its light conversion efficiency, local regions of increased forward current (local shunts) are degrading the cell performance. These regions may be detected and investigated by lock-in contact thermography allowing the detection of local current densities as low as $100 \mu \mathrm{A} / \mathrm{cm}^{2}$. This technique is described and experimentally compared with two types of IR-based measurements. We find that the detection sensitivity of the IR-measurements is at least one order of magnitude worse than that of contact thermography, as long as no high-sensitive focal plane array IR-camera and/or longer integration times can be used.
\end{abstract}

\section{Introduction}

Although solar cells have been commercialized for many years, their contribution to energy production is still negligible owing to their high price and insufficient energy conversion efficiency. Therefore, the development of highly efficient solar cells which can be produced with low costs is of great importance. These developments require analytical techniques to study the detailed origins of low solar cell efficiencies. The output power of a solar cell is the product of three basic parameters, namely the short circuit current (Jsc), the open circuit voltage (Voc), and the fill factor (FF), which is the maximum possible output power of the cell under operation conditions divided by (Jsc $\times$ Voc). In operation a solar cell becomes forward biased. While Jsc is measured under zero bias, both Voc and the FF are measured under forward bias, hence both parameters are influenced by the forward I-V-characteristic of the solar cell. Within the solar cell there is always a balance between the photogenerated current of minority carriers (which can be assumed to be nearly bias-independent) and the biasdependent diffusion current of majority carriers. The latter one is given the I-V-characteristic of the cell measured in the dark. Thus, the I-V-characteristic of the cell under illumination can be approximated by the dark I-V-characteristic shifted by the short circuit current Jsc. Under open circuit conditions (no external current flowing, $V=V o c$ ) the generated photocurrent just equals the internal forward injection current, but also in the operation point of the cell some fraction of the photogenerated current flows back internally. The larger this fraction is, the lower is the fill factor (FF) of the cell and the lower its efficiency. Thus, in order to have solar cells of large Voc and FF, one is interested to have dark I-V-characteristics of the cells having for a given bias a dark forward current as low as possible. If there should be local sites of increased current, these "shunts" may degrade the whole I-V-characteristic of a solar cell and may be responsible for a bad FF and Voc. Dynamical (lock-in) thermography, measuring the local surface temperature modulation amplitude owing to the application of bias pulses with a certain repetition frequency, is able to map the forward current density and thus to localize the sources of a bad FF or Voc.

In the following section the I-V-characteristic of real solar cells is discussed. Several techniques to image local inhomogeneities of the forward current of solar cells are introduced in chapter 3. Thereby the realization of Dynamical Precision Contact Thermography (DPCT) as the hitherto most successful technique for this purpose is briefly reviewed. In section 4 experimental results of this technique are compared with dynamical infrared thermography of 
solar cells using both an infrared camera and a $\mathrm{HgCdTe}$ IR-detector and mechanical position scanning.

\section{The I-V-characteristics of real solar cells}

According to classical diode theory a general expression for describing the I-V-characteristic of a pn-junction is given by

$$
J(V)=J_{0}\left(\exp \left(\frac{e V}{n k T}\right)-1\right)
$$

where $V$ is the applied bias, $J_{0}$ is the reverse saturation current, $e$ is the electron charge, and $k T$ is the thermal energy. The so-called ideality factor $n$ is unity as long as the current is a pure diffusion current and is not governed by recombination centres in the space charge layer. If recombination centres are involved, $\mathrm{n}$ may vary between 1 and 2 depending on the electronic parameters of the recombination centres. Exponential characteristics with an ideality factor above $n=2$ are not able to be explained in this classical diode theory. Also, dislocations crossing the pn-junction only have been shown to yield an additional current contribution according to eq. (1) with $\mathrm{n}=2$ [1]. On the contrary, measured I-V-characteristics of multicrystalline silicon solar cells typically show an exponential component having an $n$-factor in the order of $n=2.5$ [2]. At lower voltages often a linear (ohmic) current contribution appears. There have been attempts to interprete ideality factors above $n=2$ by regarding saturation effects of the involved recombination centres [3] which, however, only predicts the existence of "humps" in the I-V-characteristics. Obviously real I-V-characteristics of solar cells are hardly interpretable assuming homogeneous current flow across the cell area. On the other hand, it was shown that exponential characteristics with ideality factors well above $n=2$ may appear whenever charged extended defects having a certain interface defect density are penetrating the pn-junction, leading to non-linear local shunts in these positions [2]. Also the linear current contributions in real I-V-characteristics can hardly be explained by any theory assuming laterally homogeneous current flow, but may easily be explained assuming the existence of local shunts of ohmical type. Thus, real I-V-characteristics of multicrystalline solar cells strongly hint to the existence of local shunts in solar cells.

\section{Dynamical (lock-in) thermography on solar cells}

The most straightforward attempt to investigate inhomogeneous current flows in solar cells in a non-destructive way would be to apply a bias to it and to image the surface temperature under stationary conditions with an infrared (IR) camera as Martinuzzi et al. [4] have done. Thereby especially for silicon cells the following experimental problems appear. Owing to the good thermal conductivity of silicon the locally generated heat spreads away from the shunt position leading to a serious degradation of the spatial resolution. This lateral heat spreading may be suppressed by mounting the cell on top of a heat sink. Then, on the other hand, the local temperature contrast for usual injection densities in forward direction becomes too small to be detected using conventional IR-thermography. Note that, assuming homogeneous injection, the usual working point of a solar cell corresponds to an internally injected current density of about $2 \mathrm{~mA} / \mathrm{cm}^{2}$ at $0.5 \mathrm{~V}$ bias, corresponding to a dissipated power density of only 1 $\mathrm{mW} / \mathrm{cm}^{2}$. If the heat drain across the back of the cell would be adjusted to allow a spatial resolution of about $3 \mathrm{~mm}$, this power density would lead to a temperature increase of about one mK. Therefore, Simo and Martinuzzi [4] have not been working under forward bias but under reverse bias conditions, where many volts may be applied to the cell and the temperature contrasts may come into the order of Kelvins. However, under reverse bias only linear shunts appearing both in forward and in reverse direction may be detected. However, it had been shown that many shunts are highly non-linear, hence they appear either only under forward or only under reverse bias conditions [5]. Hence, carrying out thermography only under reverse bias may give misleading results. Therefore, for a reliable investigation of shunts under 
forward bias conditions in a non-destructive way the sensitivity of thermographic methods has to be improved.

Dynamical (lock-in) thermography has been shown by Busse et al. [6] not only to improve the sensitivity of thermal investigations but also to provide new physical effects for investigating thermal properties of materials. In this technique the sample is heated not stationary but rather sinusoidally by absorbing light irradiation with a certain repetition frequency $f$. The periodical modulation of the surface temperature is observed. The heat spreading within the material may be described by the thermal wave concept. One of the characteristics of thermal waves is the extremely strong natural damping of their amplitude along their travelling path. For a plane thermal wave of a frequency of $3 \mathrm{~Hz}$ in silicon, for example, the thermal wavelength is about $20 \mathrm{~mm}$, but already after $3 \mathrm{~mm}$ distance the amplitude reduces to $1 / \mathrm{e}$ of its initial value. Hence, at a distance of one wavelength the wave amplitude is damped by a factor of about 526 ! This strong natural damping greatly improves the spatial resolution of dynamical thermographic investigations compared to stationary ones. Lock-in thermography results may be considered both in terms of the temperature modulation amplitude signal and in terms of the phase signal of the temperature modulation relative to the phase of the exciting power. While for lock-in thermography on materials using optical excitation the inhomogeneous thermal properties of the sample are of primary interest [6], for investigating solar cells their thermal properties can be regarded as homogeneous, but the excitation intensity is the interesting inhomogeneity. Therefore, while in the first case the phase signal may be the more interesting one, for investigating solar cells the amplitude signal contains the basic information of interest. Another difference between lock-in thermography on materials and on solar cells is that in the latter case the signal behaves not linearly with respect to the excitation intensity. While for material investigations the tempeature modulation signal height always may be increased by increasing the intensity of the absorbed light, the injection current very non-linearly depends on the bias pulse height. Increasing the injection current not only shifts the working point of the cell away from that of the usual operation, but it also causes unwanted Joule heating and voltage drops at series resistances. Therefore, for investigating solar cells it is not practicable to increase the signal height by increasing the injection current. Finally, for investigating solar cells a square wave pulsed bias has to be applied as excitation instead of the sinusoidal light excitation for material investigations. The reason is that one is interested to stay on a well-defined point of the I-V-characteristic of the cell corresponding to a fixed bias. Nevertheless the dominating thermal wave is that of the basic harmonic because the system acts like a low pass filter suppressing higher harmonics. Assuming a homogeneously dissipated power density of $1 \mathrm{~mW} / \mathrm{cm}^{2}$ for a lock-in frequency of 3 $\mathrm{Hz}$ (leading to a spatial resolution of $3 \mathrm{~mm}$ ), the expected temperature modulation amplitude is about one $\mathrm{mK}$, the same as estimated above for the stationary case. However, since for this a.c. measurement temperature drifts cancel out and the signal may be integrated over a number of measurement periods, this temperature modulation is much easier to be measured than the stationary thermal contrast. At local shunts, of course, the expected temperature modulation amplitude gets correspondingly higher. As long as the shunt does not seriously short circuit the local bias of the cell, the local temperature modulation signal can be interpreted to be directly proportional to the local current density.

There are several possibilities to establish lock-in thermography on solar cells. In Fig. 1 (a-c) three measurement variants are sketched which have been compared under similar conditions in this contribution. Fig. 1 (a) shows the arrangement which is used for lock-in thermography on materials by Busse et al. [6]. It uses a conventional infrared camera (AGEMA 900, 8-12 $\mu \mathrm{m}$ ) and the commercial lock-in thermography hard- and software provided by AGEMA. Instead of the usual sinusoidal light excitation a square wave bias pulse excitation at a frequency of 1.87 $\mathrm{Hz}$ was used. This system allows to capture images of $272 \times 136$ pixels with a picture repetition frequency of about $15 \mathrm{~Hz}$ and to integrate over 1000 images, hence the total measurement time was about 1 minute. The camera was set in its highest sensitive mode (measuring from $-30^{\circ} \mathrm{C}$ to $+80^{\circ} \mathrm{C}$ ), and the image is read out with an accuracy of 12 Bit. The AGEMA hard- and software allows to carry out the lock-in procedure and delivers both the amplitude- and the 
phase-image. As an alternative technique the arrangement shown in Fig. 1 (b) has been used. Here a liquid nitrogen cooled $\mathrm{HgCdTe}$ infrared detector with germanium optics made by Infrared Associates has been used for infrared detection, and the sample was scanned mechanically using a mechanical scanner (isel-EP 1090). A pulse repetition frequency of $3 \mathrm{~Hz}$ and an integration over 5 excitation pulses per position has been used so that the measurement time per pixel was about 2 seconds. Thus, taking a $100 \times 100$ pixel image needed a measure time of about 5.5 hours. The signal of the IR detector was conditioned using a $3 \mathrm{~Hz}$ narrow-band filter and digitized by a 12 Bit $A D C$ at four equidistant times $t_{1} \ldots t_{3}$ during each excitation period. The phase-independent amplitude signal was calculated using the formula which is also used for the lock-in evaluation in the AGEMA system [6]

$$
\Delta T=\sqrt{\left(T\left(t_{1}\right)-T\left(t_{3}\right)\right)^{2}+\left(T\left(t_{2}\right)-T\left(t_{4}\right)\right)^{2}}
$$

These two lock-in thermography techniques based on the detection of the emitted infrared radiation have been compared with the Dynamical Precision Contact Thermography (DPCT) technique which is sketched in Fig. 1 (c) [5]. This technique had been developed to provide higher detection sensitivity, since a direct mechanical contact promises a better thermal contact to any kind of detector than thermal radiation can do. In order to have a high sensitivity a thermistor (K19, Siemens Matsushita) has been used as a detector rather than a thermocouple. This thermistor works in a T-measurement bridge fed by a $1 \mathrm{kHz}$ sine signal, and the bridge deflection is monitored using lock-in detection at $1 \mathrm{kHz}$. This a.c. measurement of the sensor resistance provides a higher sensitivity than a d.c. measurement of it, and it prevents that the bias pulses applied to the sample capacitively couple into the temperature detection path. The scanner is mounted within a closed box in order to protect the measurement against air-draught. In order to have a well-defined mean temperature of the sample, the solar cell is mounted on a thermostated base. A $5 \mu \mathrm{m}$ plastic foil presses the sample to this base by a vacuum and protects the sensor from direct contact to the sample. As for the previous technique the sample is scanned mechanically using the isel-EP 1090 scanner, and in each position the sensor is pressed to the sample with a load of approx. $1 \mathrm{~g}$. After positioning the sensor the system has to wait for 2 seconds until thermal equilibrium is reached, and then the actual measurement is done using 3 bias pulses with a repetition frequency of $3 \mathrm{~Hz}$. Thus, about 3 seconds measure time are needed per pixel, hence capturing a $100 \times 100$ pixel image with this technique takes nearly $10 \mathrm{hrs}$.

\section{Experimental results}

The sample used in all cases was a commercial multicrystalline silicon solar cell of $10 \times 10$ $\mathrm{cm}^{2}$ size. The measurements have been done by applying a forward bias of $0.55 \mathrm{~V}$ leading to a forward current of about $0.2 \mathrm{~A}$, which roughly corresponds to the usual operation condition of the cell. In some cases also investigations at $1 \mathrm{~A}$ forward current have been carried out in order to get a higher signal. Note, however, that according to the above discussion this current is already too high to get quantitatively interpretable results. The results of all measurements are collected in Figs. 2 (a-f). When measured with the IR-camera according to Fig. 1 (a) at a forward current of $200 \mathrm{~mA}$ the signal was totally embedded in noise. Fig. 2 (a) shows the IRcamera result measured at $1 \mathrm{~A}$ forward bias. The two bright spots at the top are the positions where the current was fed in, hence they are caused by the contact resistance. The bright spot at the left edge is a real shunt existing at the edge of the sample and showing a temperature modulation amplitude of about $30 \mathrm{mK}$. The noise level in Fig. 2 (a) is in the order of $15 \mathrm{mK}$ so that only this dominant shunt may be detected. The thermogram of the same cell measured also at $1 \mathrm{~A}$ using the $\mathrm{HgCdTe}$ detector and mechanical scanning (see Fig. 1 (b)) is shown in Fig. 2 (b). Here the whole cell is visible including several shunting regions besides that at the left edge. The current was fed to the sample at the upper right part of it. Fig. 2 (c) shows the result of the same measurement using $0.2 \mathrm{~A}$ forward bias (near operation point). Here the current feed positions are not visible anymore and besides the main shunt at the left there are still more shunts visible, but within the cell only noise can be detected. The noise level of this 
measurement can be estimated to be about $2 \mathrm{mK}$. Figs. 2 (d-f) are different representations of the result of one and the same measurement of this cell using DPCT at $0.2 \mathrm{~A}$ forward bias according to Fig. 1 (c). Fig. 2 (d) shows the result in linear grey scale between 0 and $40 \mathrm{mK}$. It shows that besides the shunts visible in Fig. 2 (c) there are even more shunts at the edge and one within the area at the upper right. If the result is displayed in linear grey scale between 0 and $4 \mathrm{mK}$ ( Fig. 2 (e)) still more shunting regions become visible both at the edges and in the interiour of the cell, but the dominant shunts appear overexposed. The noise level of this measurement is about $0.1 \mathrm{mK}$ corresponding to a current density of about $100 \mu \mathrm{A} / \mathrm{cm}^{2}$, well above the background injection density. The best representation of the result is possible by using a logarithmical gray scale (Fig. $3(f)$ ) allowing both the display of inhomogeneities within the area and the sharp localization of local shunts.

\section{Conclusions}

Fig. 2 shows that the only technique hitherto being able to image not only the dominant shunts but also background inhomogeneities of the forward current in solar cells at their operation point is Dynamical Precision Contact Thermography (DPCT). However, this is also the slowest technique needing about $10 \mathrm{hrs}$ for one measurement, and it has the disadvantage to be a contacting technique. Mechanically scanning a HgCdTe detector is a non-contact technique, but it doesn't seriously save time and it is less sensitive than DPCT by an order of magnitude. Though in this investigation lock-in thermography using an IR-camera showed the worst signal-to noise ratio, it is the most promising technique, because it requires only 1 minute measure time. Since this system was not optimized to detect smallest temperature modulations there is still room for further improvements. So the primary detection range of the camera may electronically be reduced e.g. by a factor of 10 from $-30 \ldots+60^{\circ} \mathrm{C}$ to $+20 \ldots+29^{\circ} \mathrm{C}$. Moreover, the digitizing depth may be increased from 12 to 16 Bit and, especially, the integration time should be enhanced from now $1 \mathrm{~min}$. to about $1 \mathrm{hr}$. Also increasing the optical emissivity by, e.g., wetting the sample with an oil film could improve the sensitivity of this measurement. Assuming that under optimum conditions the signal-to-noise ratio improves with the square root of the number of measurements, after an integration time of $1 \mathrm{hr}$ we expect with our IR-camera (having a nominal resolution of $80 \mathrm{mK}$ ) a temperature resolution of about $0.7 \mathrm{mK}$. If a focal plane array camera with a repetition rate of $50 \mathrm{~Hz}$ and a nominal resolution of $20 \mathrm{mK}$ would be used, after an integration time of $1 \mathrm{hr}$ the sensitivity would improve to 100 $\mu \mathrm{K}$. This is just the sensitivity obtained by DPCT, but it would be non-contacting and obtained at $1 / 10$ th of the measure time. Thus, using a focal plane array camera in an optimized lock-in thermography system should be the optimum technique to investigate the homogeneity of the forward current flow in solar cells.

\section{Acknowledgements}

This work was supported by the BMBF under contract No. $0329536 \mathrm{E}$ and, in part, by the International Soros Science Education Program (ISSEP) through grant No. PSU 062050.

\section{REFERENCES}

[1] EL GHITANI (H.), PASQUINELLI (M.), SIMON (J.J.), and MARTINUZZI (S.). - About the electrical conductibility of dislocations in multicrystalline silicon solar cells. Solid State Phenomena Vols. 37-38, 1994, p. 31-34.

[2] BREITENSTEIN (O.) and HEYDENREICH (J.). - Non-ideal l-V-characteristics of block-cast silicon solar cells. Solid State Phenomena Vols. 37-38, 1994, p. 139-144.

[3] BEIER (J.) and VOSS (B.). - Humps in dark I-V-curves - analysis and explanation. 23rd IEEE PVSC, Louisville, May 10-14, 1993, p. 321-326. 


\section{http://dx.doi.org/10.21611/qirt.1996.063}

[4] SIMO (A.) and MARTINUZZI (S.). - Hot spots and heavily dislocated regions in multicrystalline silicon cells. 21st IEEE PVSC, Kissimimee, May 21-25, 1990, p. 800-805

[5] BREITENSTEIN (O.), EBERHARDT (W.), and IWIG (K.). - Imaging the local forward current density of solar cells by dynamical Precision Contact Thermography. 1st WCPEC, Hawaii, December 5-9, 1994, p. 1633-1636.

[6] BUSSE (G.), WU (D.), and KARPEN (W.). - Thermal wave imaging with phase sensitive modulated thermography. J. Appl. Phys. 71, 1992, p. 3962-3965.
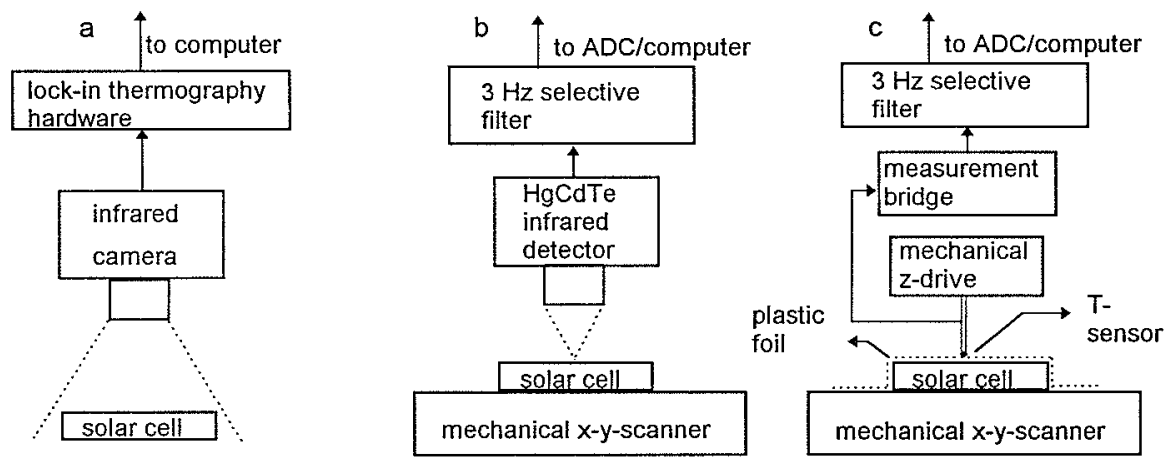

Fig. 1. Three variants of lock-in thermography of solar cells: (a) with IR camera, (b) with HgCdTe detector and mechanical scanning, and (c) contact measurement and mechanical scanning (DPCT)
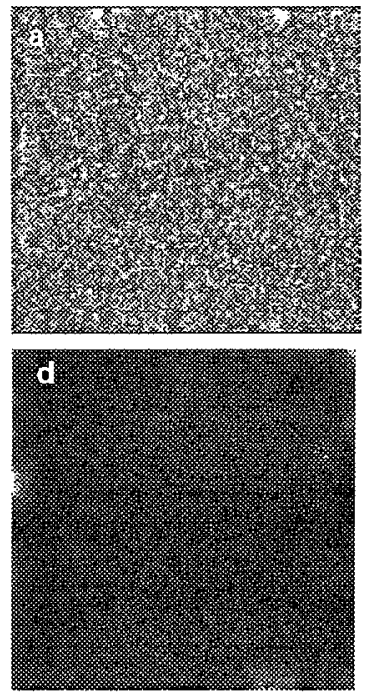
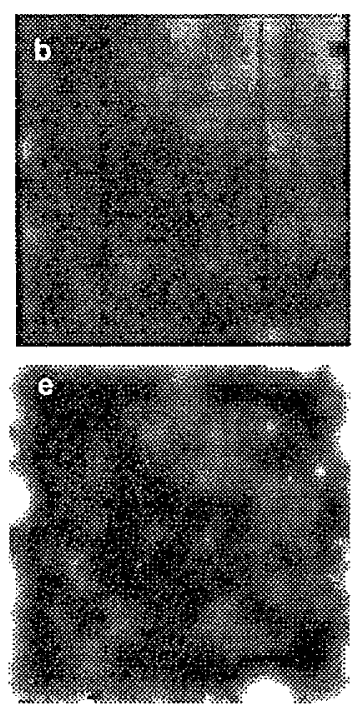
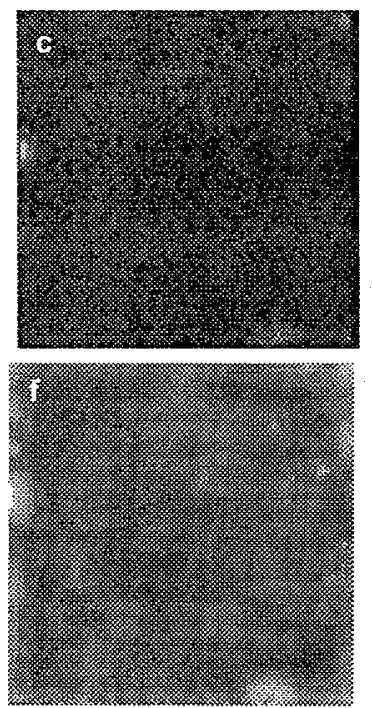

Fig. 2. Lock-in thermograms of a solar cell at different forward currents using different techniques: (a) 1A, with IR-camera (Fig. 1(a)), (b) 1A with HgCdTe detector (Fig. 1(b)), (c) 0.2A with HgCdTe detector, (d) $0.2 \mathrm{~A} \mathrm{DPCT}$ (Fig. 1(c)) $0 \ldots 40 \mathrm{mK}$, (e) $0.2 \mathrm{~A}$ DPCT $0 \ldots 4 \mathrm{mK}$, (f) $0.2 \mathrm{~A}$ DPCT log. scale 